\title{
Angular Momentum Evolution of Young Solar-type Stars
}

\author{
Louis Amard ${ }^{1,2}$, Ana Palacios ${ }^{1}$, Corinne Charbonnel ${ }^{2,3}$ \\ ${ }^{1}$ LUPM,Université Montpellier, CNRS, Place Eugène Bataillon, 34095 Montpellier France \\ email: louis . amard@univ-montp2.fr \\ ${ }^{2}$ Geneva Observatory, University of Geneva, Chemin des Maillettes, 51, 1290 Versoix, \\ Switzerland \\ ${ }^{3}$ IRAP departement of astronomy, CNRS UMR 5277, Université de Toulouse, 14, Av. E.Belin, \\ 31400 Toulouse, France
}

\begin{abstract}
We present stellar evolution models of young solar-type stars including self consistent treatment of rotational mixing and extraction of angular momentum (AM) by magnetized wind including the most up-to-date physic of AM transport.
\end{abstract}

Keywords. stars: evolution, stars: rotation, stars: wind-braking, hydrodynamics

\section{Introduction}

During their early evolution, solar-type stars undergo a considerable modification of their AM distribution due mainly to interaction with their environment. As the stillaccreting star is surrounded by its disc, the extraction of AM is mainly driven by jets and accretion; but once the star is spinning freely, the major part of the AM is removed at the surface by stellar winds outflowing along magnetic field lines while the rest is redistributed in the radiative region by hydrodynamical instabilities.

Here we use two specific prescriptions with different initial conditions to describe the impact of both phenomena (torque and internal transport of AM) on the evolution of surface and core rotation. We show in particular that rotation periods observed in open clusters over a broad age range can be reproduced with a weak core-envelope coupling using the most recent braking law available in the literature.

\section{Physics input to the stellar models}

We compute stellar models $\left(1 M_{\odot}, Z=0.0134\right)$ with the STAREVOL code (Siess et al. 2000, Palacios et al. 2006, Lagarde et al. 2012) including suitable physics for this range of mass. We account for an atmosphere with a $T-\tau$ relation following Krishna-Swamy (1966) that has been extensively tested on stellar models and gives good results (e.g. Vandenberg et al. 2007).

The treatment of convection is based on the classical mixing-length formalism with the parameter $\alpha_{M L T}=l / H_{P}=1.607$ calibrated on a solar model. Convective areas are instantaneously mixed and assumed to rotate as solid bodies. Moreover, we account for hydrostatic effects through centrifugal forces entering the hydrostatic equation following Endal \& Sofia (1976).

We follow the formalism of Zahn (1992), Maeder \& Zahn (1998) and Mathis \& Zahn (2004) to compute the secular transport of AM in the radiative zone.

$$
\rho \frac{\mathrm{d}}{\mathrm{d} t}\left(r^{2} \Omega\right)=\frac{1}{5 r^{2}} \frac{\partial}{\partial r}\left(\rho r^{4} \Omega U_{r}\right)+\frac{1}{r} \frac{\partial}{\partial r}\left(r^{4} \rho \nu_{v} \frac{\partial \Omega}{\partial r}\right)+\tau_{W}
$$


with the vertical shear diffusion coefficient $\nu_{v}$ explicited in Talon \& Zahn (1997) and the horizontal diffusion coefficient from Mathis et al. (2004). An extensive study of internal mixing formalism is done in Amard et al. (in prep.) with other prescriptions. We do not account for additional processes such as magnetic fields or internal gravity waves, which are also expected to transport AM very efficiently in the radiative zone.

The last term $\tau_{W}$ represents the AM loss due to the stellar-wind torque and is only applied at the external boundary layer. Here we present model predictions using Matt et al. (2015) prescription (other braking laws studied in Amard et al. in prep.) :

$$
\begin{gathered}
\tau_{W}=-\mathcal{T}_{0}\left(\frac{\tau_{C Z}}{\tau_{C Z \odot}}\right)^{p}\left(\frac{\Omega_{\star}}{\Omega_{\odot}}\right)^{p+1} \geqslant \text { unsaturated }, \\
\tau_{W}=-\mathcal{T}_{0} \chi^{p}\left(\frac{\Omega_{\star}}{\Omega_{\odot}}\right) \geqslant \text { saturated }
\end{gathered}
$$

with $p=1.7$ and the empirically calibrated scaling factor $\mathcal{T}_{0}$ as in Matt et al. (2015) (see also Amard et al. in prep.). This prescription accounts for a magnetic field saturation reached for $\chi=\frac{R o \odot}{R o_{\text {crit }}}=10$ with $R o_{\odot}=\left(\tau_{C Z} \odot \Omega_{\odot}\right)^{-1}$ the solar Rossby number and $R o_{\text {crit }}$ the critical one, $\tau_{C Z}$ being the convective turnover timescale in the stellar envelope.

\section{Rotation distribution}

In order to constrain the evolution, we need some quite accurate ages and only associations or open clusters can provide this accuracy. Young associations have usually too few members in a specific mass range, so we only use open cluster members with a mass between $0.9 M_{\odot}$ and $1.1 M_{\odot}$. We use the sample gathered by Gallet \& Bouvier (2015 and references therein) which includes clusters with ages between 2 Myr (ONC, NGC6530,...) and 2.5 Gyr (NGC6819). We also include the sun to constrain long-term evolution of the rotation of solar-type stars. Observations are shown in Fig. 1, where we also indicate the $90 \mathrm{th}, 50 \mathrm{th}$ and 25 th percentiles for each selected cluster computed using the rejection method (see Gallet \& Bouvier 2013). We assume that these three statistical samples represent typical evolutions of the surface rotation of young solar-type stars and these are the data we aim to reproduce with our models in the present study.

\section{Evolution of surface rotation}

We follow the evolution as soon as the star has shrinked enough to ensure a structural cohesion (surface velocity below the break-up speed). The one solar mass model is computed with three different initial velocities corresponding to the three evolutions (fast-median-slow).

Following the observations, we assume a constant angular velocity during the disccoupling phase which has a duration which is adapted accordingly: 3 Myr for the fast rotating model and $5 \mathrm{Myr}$ for the median and slow ones.

We account for AM extraction as soon as the stars are decoupled from their disc. Between the end of the disc-coupling phase and the zero-age main sequence (ZAMS), the stars are contracting and contraction dominates the evolution of surface angular velocity for the first tens of Myr. At the arrival on the ZAMS, the stars stop contracting and the surface velocities reach a maximum before the torque becomes dominant and starts spinning down the star.

The spin-down phase is driven by the internal transport of AM. AM redistribution depends on the spinning rate and on the evolution of the magnetic field strength which itself is related to the Rossby number which itself depends on the stellar surface velocity. 


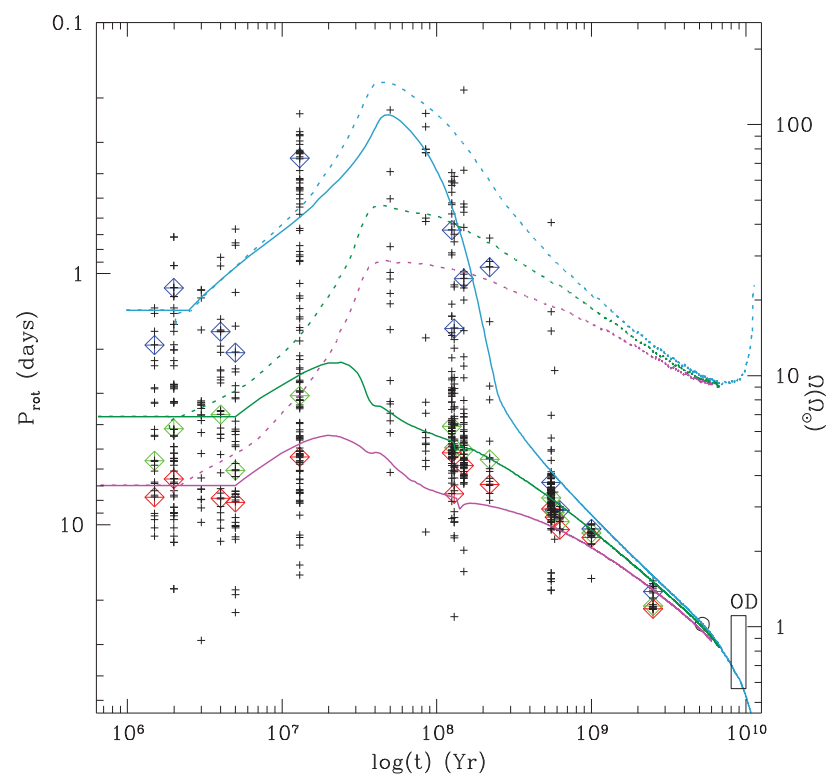

Figure 1. Evolution of angular velocities (right) and rotational periods (left) as a function of time. Crosses show stars belonging to open clusters at various ages (Gallet \& Bouvier 2015). Red, green and blue tilted squares represent respectively the 25th, 50th and 90th percentile associated to each type of rotator. Solid lines show the evolution of surface velocity for slow (magenta), median (green) and fast rotators (blue). Dashed lines represent the angular velocity integrated over the whole radiative region of each model. The open circle is the angular velocity of the present Sun and the OD black rectangle shows the angular velocity dispersion of old disk field stars (McQuillan et al. 2013).

Figure 1 presents the theoretical evolution of surface rotation for our three models (slow, moderate, and fast rotation). Predictions do well reproduce the observed rotational periods at each cluster. We can highlight two important points:

(1) After the ZAMS, surface rotation velocity is dominated by the wind-induced torque, which drives internal transport of AM. The effects of internal transport of AM on surface rotation are erased by the wind-induced torque. The latter is indeed completely dominating the evolution of the rotational period at evolutionary time-scale. But during the PMS, fast rotation and contraction induce a very strong meridional circulation spinning up the star and counter-balancing the torque.

(2) The coupling between the radiative core and the envelope of the star depends here on the surface angular velocity of the star: the fast rotator is almost in solid-body rotation until it reaches the ZAMS while slower rotators are fully decoupled, their core spinning more than 10 times faster than the surface at the arrival on the ZAMS. This can be explained by two factors: AM internal transport efficiency depends on the velocity of the star and the torque is also stronger (proportionally to the AM content) when the stellar dynamo is in a non saturated regime. Therefore, slow rotators are below the saturation limit and the surface experiences a relatively strong braking while the radiative part is accelerating freely. We can probe these effects by looking at the maximal rotation rate reached at the ZAMS compared to the initial velocity: in the case of both slow and median rotators, this maximum happens a few ten Myr earlier than for the fast rotator case. This is due to cumulative effects of weak core-envelope coupling and strong torque. In parallel, the radiative core acceleration is comparable in every case and the mean velocity is proportional to the initial velocity. 
Finally, the surface velocities of the three models converge beyond $500 \mathrm{Myr}$ to reach the solar value at the solar age following a Skumanich-like trend. In parallel, we also observe the convergence of the integrated angular velocities in the radiative zone. This indicates that at this phase, all our models experience the same efficiency for the transport of AM independently of the initial conditions. Turning to other indicators such as the light elements surface abundances would help to disentangle between these three different evolutions.

\section{Conclusions}

We present stellar evolutionary models including a self-consistent treatment of rotationinduced mixing and realistic AM extraction allows us to study the effect of different physical processes on stellar rotation along the evolution. We show that including only hydrodynamical mechanisms we can reach a unique rotation profile at the age of the Sun independent of the initial angular momentum content, losing memory of all previous evolution.

The surface angular velocity evolution essentially constrains the torque. The latter dominates the integrated surface rotation evolution but is not constraining the internal AM transport. Indeed, asteroseismology (e.g. Garcia et al. 2011) and stellar chemical properties (e.g. Bouvier 2008) reveal a flattened profile for the actual Sun in opposition to the important core-envelope decoupling we obtain in our model. This can be explained by additional processes such as internal gravity waves (Charbonnel et al. 2013, Charbonnel \& Talon 2005) or magnetic fields (Eggenberger et al. 2005), which appear to be very good candidates to transport efficiently AM in the radiative zone. The next step will be to include those processes in the computation to test their efficiency with a realistic torque.

\section{References}

Amard, L., Palacios, A., Charbonnel, C. in prep

Bouvier, J. 2008, A\& A, 489, 53

Charbonnel, C., Decressin, T., Amard, L., Palacios, A., \& Talon, S. 2013, A\&A, 554, A40

Charbonnel, C. \& Talon, S. 2005, Science, 309, 2189

Eggenberger, P., Maeder, A., \& Meynet, G. 2005, A\&A, 440, L9

Endal, A. S. \& Sofia, S. 1976, ApJ, 210, 184

Gallet, F. \& Bouvier, J. 2013, A\&A, 556, A36

Gallet, F. \& Bouvier, J. 2015, A\&A, 577, A98

García, R.-A., Salabert, D., \& Ballot, J. et al. 2011, JPhCS, 271

Krishna-Swamy, K. S. 1966, ApJ 145,174

Lagarde, N. \& Decressin, T., Charbonnel C. et al. 2012, A\&A, 543, 108

Maeder, A. \& Zahn, J.-P. 1998, A\&A, 334, 1000

Mathis, S., Palacios, A., \& Zahn, J.-P. 2004, A\&A, 425, 243

Mathis, S. \& Zahn, J.-P. 2004, A\&SA, 425, 229

Matt, S., Brun, A. S., Baraffe, I., Bouvier, J., \& Chabrier, G. 2015, ApJ, 799, L23

McQuillan, A., Mazeh, T., \& Aigrain, S. 2013, ApJL, 775, L11

Palacios, A., Charbonnel, C., Talon, S., \& Siess, L. 2006, A\&A, 453, 261

Siess, L., Dufour, E., \& Forestini, M. 2000, A\& $A$, 358, 593

Talon, S. \& Zahn, J.-P. 1997, A\&̈A, 317, 749

Vandenberg, D. A., Edvardsson, B., Eriksson, K., Gustafsson, B., \& Ferguson, J. W. 2007, in IAU Symp. 241, Stellar Populations as Building Blocks of Galaxies, p. 23

Zahn, J.-P. 1992, A\&A, 265, 115

\section{Discussion}

Pinsonneault: Comment: K2 mission is about to get asteroseismic data from young stars around 30 to $100 \mathrm{Myr}$ allowing to potentially probe internal rotation during this crucial phase. 